\title{
Optimal OFDM Pilot Sequences for Time-Delay and Channel Estimation Based on the Expected CRB for a Large Number of Subcarriers
}

\author{
Arash Shahmansoori, Rafael Montalban and Gonzalo Seco-Granados \\ Universitat Autònoma de Barcelona, \\ Bellaterra, Barcelona 08193, Spain \\ \{arash.shahmansoori, rafael.montalban, gonzalo.seco\}@uab.cat
}

\begin{abstract}
A key aspect to design an OFDM system for combined positioning and high-data-rate communications is to find optimal data and pilot power allocations. Previous work has investigated the capacity maximizing design taking into account the effects of channel and time-delay estimation for finite number of subcarriers and channel taps. In this paper, we propose a method based on the asymptotic expected Cramér-Rao bound of joint time-delay and channel coefficients that reduces the complexity of the bounds by increasing the number of subcarriers or channel taps for data and pilot power allocations design. Specifically, for long channels a general form of matrix inversion, which is computationally complex, is converted to only the inversion at strong eigenvalues or pilots. Numerical results show that as the number of subcarriers increases, the non-asymptotic bounds converge to the asymptotic bounds at a fast speed. Moreover, even for a finite number of subcarriers or channel taps the difference between joint data and pilot power allocations is negligible compared to the non-asymptotic expected Cramér-Rao bounds.
\end{abstract}

Index Terms-Combined positioning and communications, OFDM, channel and time-delay estimation, asymptotic expected Cramér-Rao bound, joint design of data and pilot power allocations.

\section{INTRODUCTION}

The design of combined positioning and communications systems that can perform well in terms of high-data-rate transmission and estimation accuracy is a challenging problem. In general, the signals used for one application perform poorly in the other case. To design a signal which can be applied for both purposes, one needs to consider the system specifications for time-delay estimation accuracy and data-rate communications.

To date, different approaches have been adopted to design pilot symbols that improve the performance of channel estimators [1]-[3]. The results show that equi-spaced, equi-powered pilots

This work was financially supported by EU FP7 Marie Curie Initial Training Network MULTI-POS (Multi-technology Positioning Professionals) under grant nr. 316528.

This work was supported by R\&D Project of Spanish Ministry of Economy and Competitiveness TEC2011-28219. are optimal in terms of mean squared error. Pilot designs for carrier frequency offset (CFO) estimation [4], or joint channel and CFO estimation [5]-[7] are considered by others. However, pilot design based on time-delay estimation has received little attention. A pilot design based on joint CRB of channel and time delay is proposed in [8], [9]. However, since CRBs in [8], [9] are functions of specific channel realizations, the resulting pilots cannot be guaranteed to be optimal for all instances of random channels. The problem has been solved by designing based on averaging the CRB over a set of channel realizations known as Expected CRB (ECRB) [10]. Furthermore, joint data and pilot power allocations for the case of limited number of subcarriers $N$ and channel taps $L$ is investigated [10]. A method based on the effect of increasing the number of subcarriers $N$ and channel taps $L$ on joint channel coefficients and clock offset estimation is proposed in [11]. However, the bounds are limited to a specific type of channel.

In this paper, we consider the effect of increasing the number of subcarriers $N$ and channel taps $L$ on the joint expected Cramér-Rao bound of time-delay and channel coefficients. Using asymptotic bounds reduce the computational complexity. Specifically, applying matrix inversion algorithms such as Gaussian elimination requires the computational complexity of the order $O\left(L^{3}\right)$ for non-asymptotic bounds that makes non-asymptotic bounds in [10] complex and close to singular. However, we reduce the complexity to $O(L)$ by doing the inversion only at strong pilots. Asymptotic expected CramérRao bound for joint time-delay and channel coefficients is investigated for two cases. First, we assume the number of subcarriers $N$ is sufficiently large but the number of channel taps $L$ is finite, second we consider the case where $L$ and $N$ both grows large, with their relative ratio $L / N$ approaching zero. Furthermore, we compare joint design of data and pilot allocations based on asymptotic bounds with non-asymptotic bounds. Our results show that the difference for joint design of data and pilot allocations based on asymptotic bounds is negligible. 


\section{System Model AND PReliminaries}

In this section, first we present a model of the OFDM system, then we propose the ECRB of the joint timing offset and channel coefficients estimation as a performance metric.

\section{A. OFDM Signal Model}

Using the same notation as [11], the continuous-time received signal from a standard OFDM symbol passed through a frequency selective channel, after removing the guard interval, is

$$
y_{N}^{(a)}(t)=\sum_{k \in \mathbb{Z}} d_{N, k} g^{(a)}(t-k T)+v^{(a)}(t),
$$

where $T$ is the sampling period at the transmitter such that $T_{0}=N T$ is the observation window, $N$ is the total number of subcarriers, $d_{N, k}$ represents the output from the inverse FFT (IFFT) block at the transmitter, and $v^{(a)}(t)$ is additive zeromean complex Gaussian noise. Unlike [11], but without loss of generality, we assume that the impulse response $g^{(a)}(t)$ is a delta function with the time limit of $[0, L T)$ where $L$ is the number of channel taps

$$
g^{(a)}(t)=\sum_{l=0}^{L-1} h_{l} \delta(t-l T-\tau),
$$

where $h_{l}$ is the channel coefficient of $l$ th path and $\tau$ is the timing offset or equivalently the time delay of first path. Assuming the transmitter's and receiver's clocks are synchronized, the discrete-time received signal $y_{N}[n]=y_{N}^{(a)}(n T)$ is

$$
y_{N}[n]=\frac{1}{\sqrt{N}} \sum_{n^{\prime}=0}^{N-1} \sum_{l=0}^{L-1} D_{N, n^{\prime}} h_{l} e^{j \frac{2 \pi}{N} n^{\prime}\left(n-l-\tau_{d}\right)}+v[n],
$$

where $D_{N, n^{\prime}}$ represents pilot subcarrier at the $n^{\prime}$ th frequency, and $\tau=\tau_{d} T$. In vector form we have

$$
\boldsymbol{y}_{N}=\boldsymbol{R}_{N}\left(\tau_{d}\right) \boldsymbol{h}+\boldsymbol{v}_{N},
$$

where $\boldsymbol{y}_{N}=\left[y_{N}[0], \ldots, y_{N}[N-1]\right]^{T}, \quad \boldsymbol{v}_{N}=$ $\left[v_{N}[0], \ldots, v_{N}[N-1]\right]^{T}, \quad h=\left[h_{0}, \ldots, h_{L-1}\right]^{T}$, and the $(n, l)$ element of $\boldsymbol{R}_{N}\left(\tau_{d}\right)$ is $\left[\boldsymbol{R}_{N}\left(\tau_{d}\right)\right]_{n, l}=$ $\frac{1}{\sqrt{N}} \sum_{n^{\prime}=0}^{N-1} D_{N, n^{\prime}} e^{j \frac{2 \pi}{N} n^{\prime}\left(n-l-\tau_{d}\right)}$. Finally, taking the FFT of the output, we find

$$
\boldsymbol{Y}_{N}=\boldsymbol{F}_{N, N} \boldsymbol{y}_{N}=\boldsymbol{F}_{N, N} \boldsymbol{R}_{N}\left(\tau_{d}\right) \boldsymbol{h}+\boldsymbol{V}_{N},
$$

which describes the output of the OFDM system at the training phase. Also, one can obtain a similar model as in [8] by taking the DFT of (3) and writing the result in a vector notation as

$$
\boldsymbol{Y}_{N}=\boldsymbol{D}_{N} \boldsymbol{\Gamma}\left(\tau_{d}\right) \boldsymbol{F}_{N, L} \boldsymbol{h}+\boldsymbol{V}_{N},
$$

where $\boldsymbol{D}_{N}$ represents an $N \times N$ diagonal matrix of the input with the $k$ th diagonal element $D_{N, k}$ representing the input at the $k$ th subcarrier, $\boldsymbol{\Gamma}$ is an $N \times N$ diagonal matrix with the $k$ th diagonal element $\exp \left(-j 2 \frac{\pi}{T} k \tau_{d}\right)$, and $\boldsymbol{F}_{N, L}$ contains the first $L$ columns of a Discrete Fourier Transform (DFT) matrix. Note that the difference between our problem and the problems presented in [5], [11] is that here the timing offset is only multiplied by $n^{\prime}$, while in [11], sampling clock frequency offset is multiplied by both $n^{\prime}$ and $n$. Also, the problem differs from the case when frequency offset occurs [5] since in that case frequency offset is multiplied by $n$ not $n^{\prime}$.

\section{B. Expected Cramér-Rao Bound}

In this section, we present a closed-form expression for the expected CRB for the channel coefficients $\boldsymbol{h}$ and the timing offset $\tau_{d}$. Note that the related results can be found in [5], [8], [10], [11]. Defining the parameter vector by $\boldsymbol{\theta}=\left[\boldsymbol{h}_{R}^{T}, \boldsymbol{h}_{I}^{T}, \tau_{d}\right]^{T}$, the corresponding Fisher information matrix (FIM) can be written as

$$
\boldsymbol{J}_{F}=\frac{2}{\sigma^{2}} \Re\left[\frac{\partial \boldsymbol{\mu}^{H}}{\partial \boldsymbol{\theta}} \frac{\partial \boldsymbol{\mu}}{\partial \boldsymbol{\theta}^{T}}\right],
$$

where $\boldsymbol{\mu}=\boldsymbol{F}_{N, N} \boldsymbol{R}_{N}\left(\tau_{d}\right) \boldsymbol{h}$. One can easily find the FIM

$$
\boldsymbol{J}_{F}=\frac{2}{\sigma^{2}}\left[\begin{array}{ccc}
N \Re\left[\boldsymbol{U}_{N}\right] & -N \Im\left[\boldsymbol{U}_{N}\right] & N^{2} \Re\left[\boldsymbol{V}_{N} \boldsymbol{h}\right] \\
N \Im\left[\boldsymbol{U}_{N}\right] & N \Re\left[\boldsymbol{U}_{N}\right] & N^{2} \Im\left[\boldsymbol{V}_{N} \boldsymbol{h}\right] \\
N^{2} \Re\left[\boldsymbol{h}^{H} \boldsymbol{V}_{N}^{H}\right] & -N^{2} \Im\left[\boldsymbol{h}^{H} \boldsymbol{V}_{N}^{H}\right] & N^{3} \boldsymbol{h}^{H} \boldsymbol{W}_{N} \boldsymbol{h}
\end{array}\right]_{(8)}
$$

where $\boldsymbol{U}_{N}=\frac{1}{N} \boldsymbol{R}_{N}^{H}\left(\tau_{d}\right) \boldsymbol{R}_{N}\left(\tau_{d}\right), \boldsymbol{V}_{N}=\frac{1}{N^{2}} \boldsymbol{R}_{N}^{H}\left(\tau_{d}\right) \boldsymbol{Q}_{N}\left(\tau_{d}\right)$, $\boldsymbol{W}_{N}=\frac{1}{N^{3}} \boldsymbol{Q}_{N}^{H}\left(\tau_{d}\right) \boldsymbol{Q}_{N}\left(\tau_{d}\right)$, and $\boldsymbol{Q}_{N}\left(\tau_{d}\right)=d \boldsymbol{R}_{N}\left(\tau_{d}\right) / d \tau_{d}$. Using the well known block inversion matrix lemma [12] and defining a new estimation parameter $\tilde{\boldsymbol{\theta}}=\left[\boldsymbol{h}^{T}, \tau_{d}\right]^{T}$, we find

$$
\begin{gathered}
\mathbb{E}\left[\left\|\hat{\boldsymbol{h}}_{N}-\boldsymbol{h}\right\|^{2}\right] \geq \frac{\sigma^{2}}{2 N}\left(2 \operatorname{tr}\left(\boldsymbol{U}_{N}^{-1}\right)+\gamma_{N}^{-1}\left\|\boldsymbol{\beta}_{N}\right\|^{2}\right), \\
\mathbb{E}\left[\left(\hat{\tau}_{d}^{N}-\tau_{d}\right)^{2}\right] \geq \frac{\sigma^{2}}{2 N^{3} \gamma_{N}},
\end{gathered}
$$

where

$$
\begin{gathered}
\boldsymbol{\beta}_{N}=\boldsymbol{U}_{N}^{-1} \boldsymbol{V}_{N} \boldsymbol{h}, \\
\gamma_{N}=\boldsymbol{h}^{H}\left(\boldsymbol{W}_{N}-\boldsymbol{V}_{N}^{H} \boldsymbol{U}_{N}^{-1} \boldsymbol{V}_{N}\right) \boldsymbol{h} .
\end{gathered}
$$

Finally, taking the expectation with respect to channel coefficients and using Jensen's inequality, the approximate expression for the ECRB of the timing offset and the channel coefficients would be

$$
\begin{aligned}
& \operatorname{ECRB}_{h} \approx \frac{\sigma^{2}}{2 N}\left(2 \operatorname{tr}\left(\boldsymbol{U}_{N}^{-1}\right)+\bar{\gamma}_{N}^{-1}\left\|\overline{\boldsymbol{\beta}}_{N}\right\|^{2}\right), \\
& \operatorname{ECRB}_{\tau_{d}} \approx \frac{\sigma^{2}}{2 N^{3} \bar{\gamma}_{N}},
\end{aligned}
$$

where

$$
\begin{gathered}
\left\|\overline{\boldsymbol{\beta}}_{N}\right\|^{2}=\operatorname{tr}\left(\left(\boldsymbol{V}_{N}^{H} \boldsymbol{U}_{N}^{-H} \boldsymbol{U}_{N}^{-1} \boldsymbol{V}_{N}\right) \boldsymbol{R}_{h}\right), \\
\bar{\gamma}_{N}=\operatorname{tr}\left(\left(\boldsymbol{W}_{N}-\boldsymbol{V}_{N}^{H} \boldsymbol{U}_{N}^{-1} \boldsymbol{V}_{N}\right) \boldsymbol{R}_{h}\right),
\end{gathered}
$$

where $\boldsymbol{R}_{h}$ is the channel covariance matrix. Note that the actual expected CRB is tighter than the above expressions due to Jensen's inequality. In the next section, we obtain the asymptotic ECRB for channel coefficients and timing offset where the number of subcarriers $N$ is assumed to be sufficiently large. 


\section{ASYMPTOTIC EXPECTED CRAMÉR-RAO BOUND}

In this section, we obtain an asymptotic expression for $\boldsymbol{U}_{N}$, and using a similar procedure as in [11], we conclude the expressions for $\boldsymbol{V}_{N}$ and $\boldsymbol{W}_{N}$. Unlike the expressions proposed in [11], we express the asymptotic values of $\boldsymbol{U}_{N}, \boldsymbol{V}_{N}$, and $\boldsymbol{W}_{N}$ in discrete frequency as a function of pilot powers since the final goal would be to design a system with optimal power allocation for joint communication and navigation.

The $(p, q)$ element of $\boldsymbol{U}_{N}$ is

$$
\begin{gathered}
{\left[\boldsymbol{U}_{N}\right]_{p, q}=} \\
\frac{1}{N} \sum_{l_{1}, l_{2}=0}^{N-1} \boldsymbol{D}_{N, l_{1}}^{*} \boldsymbol{D}_{N, l_{2}} e^{j \frac{2 \pi}{N}\left(l_{1}-l_{2}\right) \tau_{d}}\left[\boldsymbol{M}^{H}\left(l_{1}\right) \boldsymbol{M}\left(l_{2}\right)\right]_{p, q},
\end{gathered}
$$

where

$$
\left[\boldsymbol{M}^{H}\left(l_{1}\right) \boldsymbol{M}\left(l_{2}\right)\right]_{p, q}=e^{-j \frac{2 \pi}{N}\left(q l_{2}-p l_{1}\right)} \psi_{N}^{(0)}\left(\left(l_{1}-l_{2}\right)\right),
$$

being

$$
\psi_{N}^{(0)}\left(\left(l_{1}-l_{2}\right)\right)=\frac{1}{N} \sum_{n=0}^{N-1} e^{-j \frac{2 \pi}{N} n\left(l_{1}-l_{2}\right)} .
$$

We propose the following lemma [11]:

Lemma 3.1: Let $\alpha>0$ and let $\phi_{N}: \mathbb{N} \rightarrow \mathbb{C}$ be a function such for every integer $k$ with $1<|k|<\lfloor\alpha N\rfloor,\left|\phi_{N}(k)\right|<$ $C / k$, where $C$ is a constant that does not depend on $N$. Then, for every real number $r$

$$
\psi_{N}^{(0)}\left(\left(l_{1}-l_{2}\right)\right)=\frac{1}{N} \sum_{n=0}^{N-1} e^{-j \frac{2 \pi}{N} n\left(l_{1}-l_{2}\right)},
$$

converges almost surely to 0 as $N \rightarrow \infty$.

Proof: See [11].

Replacing (18) and (19) in (17), and using the above lemma with $r=q-p+\tau_{d}, \alpha=1 / 2$ and $\phi_{N}(k)=e^{-j \frac{2 \pi}{N} p k} \psi_{N}^{(0)}(k)$, one can conclude that the terms for $l_{1} \neq l_{2}$ in (17) almost surely converge to zero. Therefore, we obtain

$$
[\boldsymbol{U}]_{p, q}=\left[\boldsymbol{U}_{N \rightarrow \infty}\right]_{p, q}=\frac{1}{N} \sum_{l=0}^{N-1}\left|D_{N, l}\right|^{2} e^{-j \frac{2 \pi}{N}(q-p) l} .
$$

Similarly, the asymptotic expressions for $\boldsymbol{V}_{N}$ and $\boldsymbol{W}_{N}$ can be found as

$$
[\boldsymbol{V}]_{p, q}=\left[\boldsymbol{V}_{N \rightarrow \infty}\right]_{p, q}=\frac{j \pi}{N} \sum_{l=0}^{N-1} \frac{l}{N}\left|D_{N, l}\right|^{2} e^{-j \frac{2 \pi}{N}(q-p) l},
$$

and

$$
[\boldsymbol{W}]_{p, q}=\left[\boldsymbol{W}_{N \rightarrow \infty}\right]_{p, q}=\frac{4 \pi^{2}}{3 N} \sum_{l=0}^{N-1}\left(\frac{l}{N}\right)^{2}\left|D_{N, l}\right|^{2} e^{-j \frac{2 \pi}{N}(q-p) l} .
$$

In the following, we find a more compact form for the asymptotic expressions of channel coefficients and time delay and also we consider the case when the number of channel taps $L$ is sufficiently large such that $L / N \rightarrow 0$. Replacing $\left|D_{N, l}\right|^{2}$ by $P_{l}$ as the pilot power at the $l$ th subcarrier, and using (21), (22), and (23) we obtain $\boldsymbol{U}=\boldsymbol{U}_{N \rightarrow \infty}=\frac{1}{N} \sum_{l=0}^{N-1} P_{l} \boldsymbol{e}(l) \boldsymbol{e}^{H}(l)$, $\boldsymbol{V}=\boldsymbol{V}_{N \rightarrow \infty}=\frac{j \pi}{N} \sum_{l=0}^{N-1} \frac{l}{N} P_{l} \boldsymbol{e}(l) \boldsymbol{e}^{H}(l)$, and $\boldsymbol{W}=$ $\boldsymbol{W}_{N \rightarrow \infty}=\frac{4 \pi^{2}}{3 N} \sum_{l=0}^{N-1}\left(\frac{l}{N}\right)^{2} P_{l} \boldsymbol{e}(l) \boldsymbol{e}^{H}(l)$ where $\boldsymbol{e}(l)=$ $\left[e^{j \frac{2 \pi}{N} l(0)}, \ldots, e^{j \frac{2 \pi}{N} l(L-1)}\right]^{T}$. This can be interpreted as the sum of $N, L \times L$ matrices with the eigenvalues $P_{l}$ and eigenvectors $e(l)$ for $l=0, \ldots, N-1$. In matrix form, we have $\boldsymbol{U}=\boldsymbol{F}_{L, N} \boldsymbol{P} \boldsymbol{F}_{L, N}^{H}, \boldsymbol{V}=j \pi \boldsymbol{F}_{L, N} \boldsymbol{D P} \boldsymbol{F}_{L, N}^{H}$, and $\boldsymbol{W}=\frac{4 \pi^{2}}{3} \boldsymbol{F}_{L, N} \boldsymbol{D}^{2} \boldsymbol{P} \boldsymbol{F}_{L, N}^{H}$ where $\boldsymbol{F}_{L, N}$ is the first $L$ rows of the discrete Fourier transform matrix, and $\boldsymbol{P}$ and $\boldsymbol{D}$ are pilot power and derivative matrices defined as $\boldsymbol{P}=\operatorname{diag}\left\{P_{0}, \ldots, P_{N-1}\right\}$ and $\boldsymbol{D}=\operatorname{diag}\left\{\frac{0}{N}, \ldots, \frac{N-1}{N}\right\}$ respectively. Consequently, asymptotic ECRB of channel coefficients and time delay can be found by replacing $\boldsymbol{U}, \boldsymbol{V}$, and $\boldsymbol{W}$ in (13) and (14),

$$
\begin{gathered}
\operatorname{NECRB}_{h}^{a s} \approx \frac{\sigma^{2}}{2}\left(2 \operatorname{tr}\left(\boldsymbol{U}^{-1}\right)+\bar{\gamma}^{-1}\|\overline{\boldsymbol{\beta}}\|^{2}\right), \\
N^{3} \mathrm{ECRB}_{\tau_{d}}^{a s} \approx \frac{\sigma^{2}}{2 \bar{\gamma}},
\end{gathered}
$$

where

$$
\|\overline{\boldsymbol{\beta}}\|^{2}=\operatorname{tr}\left(\left(\boldsymbol{V}^{H} \boldsymbol{U}^{-H} \boldsymbol{U}^{-1} \boldsymbol{V}\right) \boldsymbol{R}_{h}\right),
$$

and

$$
\bar{\gamma}=\operatorname{tr}\left(\left(\boldsymbol{W}-\boldsymbol{V}^{H} \boldsymbol{U}^{-1} \boldsymbol{V}\right) \boldsymbol{R}_{h}\right) .
$$

Finally, we analyze a special case where the number of channel taps $L$ is sufficiently large such that $L / N \rightarrow 0$. In this case the Fisher information matrix (FIM) is close to singular or badly conditioned. For this type of problem, it is proved in [13] that instead of using the inverse of the FIM, we should apply the pseudo-inverse. This means that the inverse terms in (24) and (25), i.e. $\boldsymbol{U}^{-1}$ and $\boldsymbol{U}^{-2}$, should be replaced by their pseudo-inverse. Equivalently, the corresponding eigenvectors to weak eigenvalues are set to zero. Applying eigenvalue decomposition to (24) and (25), we obtain

$$
N \overline{\mathrm{ECRB}}_{h}^{a s} \approx \sigma^{2}\left[\operatorname{tr}\left(\boldsymbol{\Lambda}^{-1}\right)+\frac{3 \operatorname{tr}\left(\left(\boldsymbol{E} \tilde{\boldsymbol{D}}^{2} \boldsymbol{E}^{H}\right) \boldsymbol{R}_{h}\right)}{2 \operatorname{tr}\left(\left(\boldsymbol{E} \tilde{\boldsymbol{D}}^{2} \boldsymbol{\Lambda} \boldsymbol{E}^{H}\right) \boldsymbol{R}_{h}\right)}\right],
$$

and

$$
N^{3} \overline{\mathrm{ECRB}}_{\tau_{d}}^{a s} \approx \frac{3 \sigma^{2}}{2 \pi^{2}} \frac{1}{\operatorname{tr}\left(\left(\boldsymbol{E} \tilde{\boldsymbol{D}}^{2} \boldsymbol{\Lambda} \boldsymbol{E}^{H}\right) \boldsymbol{R}_{h}\right)},
$$

where $\boldsymbol{E}$ represents the eigenvectors corresponding to the strong eigenvalues or pilots. Note that the terms eigenvalues and pilots have been used interchangeably since, by increasing the number of channel taps, the eigenvalues and pilots are asymptotically the same. $\tilde{D}$ is the derivative matrix at the subcarriers with strong pilots, and $\boldsymbol{\Lambda}$ represents strong eigenvalues or pilots. Note that (28) and (29) have been computed by replacing $\boldsymbol{U}^{-1}, \boldsymbol{V}$, and $\boldsymbol{W}$ by $\boldsymbol{E} \Lambda^{-1} \boldsymbol{E}^{H}, \boldsymbol{E} \tilde{\boldsymbol{D}} \boldsymbol{\Lambda} \boldsymbol{E}^{H}$, and $\boldsymbol{E} \tilde{\boldsymbol{D}}^{2} \boldsymbol{\Lambda} \boldsymbol{E}^{H}$ respectively, and using the fact that $\boldsymbol{E}$ is unitary matrix (i.e., $\boldsymbol{E}^{H} \boldsymbol{E}=\boldsymbol{I}$ ). This is pretty similar to the 
expressions proposed in [5] for the case of Asymptotic CRB of joint frequency offset and channel coefficients.

\section{ChannEl CAPACITY}

In this section we present a pilot design for joint communication and navigation based on asymptotic expected CRB for two cases. First, a more general form when the number of channel taps can be any limited number is investigated. Second, we assume sufficiently large number of channel taps $L$ such that the ratio between channel taps and the number of subcarriers goes to zero $L / N \rightarrow 0$. We can rewrite signal model (6) as

$$
\boldsymbol{Y}_{N}=\boldsymbol{H} \dot{\boldsymbol{D}}_{N}+\boldsymbol{V}_{N}
$$

where $\dot{\boldsymbol{D}}_{N}=\left[D_{N, 0}, \ldots, D_{N, N-1}\right]^{T}, \quad \boldsymbol{H}=$ $\operatorname{diag}\{H(0), \ldots, H(N-1)\}, \quad H(k)=\gamma_{\tau}(k) \bar{H}(k)$, $\gamma_{\tau}(k)=\exp \left(-j \frac{2 \pi}{T_{0}} k \tau\right), \bar{H}(k)=\boldsymbol{F}_{k, L} \boldsymbol{h}$, and $\boldsymbol{F}_{k, L}$ is the $k$ th row of $\boldsymbol{F}_{N, L}$. Replacing $\boldsymbol{H}$ by $\hat{\boldsymbol{H}}+\tilde{\boldsymbol{H}}$ in (30) we obtain

$$
\boldsymbol{y}=\hat{\boldsymbol{H}} \dot{\boldsymbol{D}}_{N}+\tilde{\boldsymbol{H}} \dot{\boldsymbol{D}}_{N}+\boldsymbol{V}_{N}
$$

where $\hat{\boldsymbol{H}}$ and $\tilde{\boldsymbol{H}}$ represent the estimated value and the error, with the $k$ th diagonal elements $\hat{H}(k)=\gamma_{\hat{\tau}}(k) \boldsymbol{F}_{k, L} \hat{\boldsymbol{h}}$ and $\tilde{H}(k)=\gamma_{\tilde{\tau}}(k) \boldsymbol{F}_{k, L} \tilde{\boldsymbol{h}}$ respectively. Note that channel coefficients $\boldsymbol{h}$ are estimated in the receiver through known pilot symbols inserted at the transmitter. The receiver feeds the estimated channel coefficients back to the transmitter (Adaptive Modulation). However, the estimated coefficients at the transmitter are not error-free due to estimation error. Consequently, it can be shown that the lower bound of ergodic capacity $\bar{C}_{l b}$ for the so called partially known channel at the transmitter is of the form of [14]

$$
\bar{C}_{l b}\left(\boldsymbol{p}_{p}, \boldsymbol{p}_{d}\right)=\frac{1}{N} \mathbb{E}\left[\log _{2} \operatorname{det}\left(\boldsymbol{I}+\boldsymbol{P}_{d} \boldsymbol{R}_{y_{e}}^{-1} \hat{\boldsymbol{H}} \hat{\boldsymbol{H}}^{H}\right)\right],
$$

where $\boldsymbol{P}_{d}$ is an $N \times N$ diagonal matrix of data power with $k \in \mathcal{D}$ diagonal entry $p_{d, k}$ and zero elsewhere, $\mathcal{D}$ being the set of subcarriers used for data transmission, and

$$
\boldsymbol{R}_{y_{e}}=\boldsymbol{P}_{d} \mathbb{E}\left[\tilde{\boldsymbol{H}} \tilde{\boldsymbol{H}}^{H}\right]+\sigma^{2} \boldsymbol{I} .
$$

So, we obtain

$$
\mathbb{E}\left[\tilde{\boldsymbol{H}} \tilde{\boldsymbol{H}}^{H}\right]=\operatorname{diag}\left\{\boldsymbol{F}_{k, L} \mathbb{E}\left\{\tilde{\boldsymbol{h}} \tilde{\boldsymbol{h}}^{H}\right\} \boldsymbol{F}_{k, L}^{H}\right\}_{k \in \mathcal{D}} .
$$

Using (33) and (34), and replacing $\mathbb{E}\left\{\tilde{\boldsymbol{h}} \tilde{\boldsymbol{h}}^{H}\right\}$ by the matrix form of (24) or (28), we find the ergodic capacity as

$$
\bar{C}_{l b}\left(\boldsymbol{p}_{p}, \boldsymbol{p}_{d}\right)=\mathbb{E}\left[C_{l b}\left(\boldsymbol{p}_{p}, \boldsymbol{p}_{d}\right)\right],
$$

where $C_{l b}\left(\boldsymbol{p}_{p}, \boldsymbol{p}_{d}\right)$ is the instantaneous capacity defined as

$$
C_{l b}\left(\boldsymbol{p}_{p}, \boldsymbol{p}_{d}\right)=\frac{1}{N} \sum_{k \in \mathcal{D}} \log _{2}\left(1+\frac{p_{d, k} \zeta_{k}}{p_{d, k} \widetilde{\zeta}_{k}\left(\boldsymbol{p}_{p}\right)+\sigma^{2}}\right),
$$

where $\zeta_{k}=\boldsymbol{F}_{k, L} \hat{\boldsymbol{h}} \hat{\boldsymbol{h}}^{H} \boldsymbol{F}_{k, L}^{H}$, and $\widetilde{\zeta}_{k}\left(\boldsymbol{p}_{p}\right)=\boldsymbol{F}_{k, L} \boldsymbol{J}_{h}\left(\boldsymbol{p}_{p}\right) \boldsymbol{F}_{k, L}^{H}$ with $\boldsymbol{J}_{h}\left(\boldsymbol{p}_{p}\right)$ being the matrix form of (24) or (28), and $\boldsymbol{p}_{p}$ represents pilot vector or subcarriers used for estimation, i.e. $\widetilde{\zeta}_{k}\left(\boldsymbol{p}_{p}\right)$ is a function of pilot vector $\boldsymbol{p}_{p}$.

\section{Power Allocation Optimization}

In this section, we formulate the optimization problem used for pilot design for joint communications and positioning. To maximize the cost function which is the lower bound of ergodic capacity (35), one needs to solve the following optimization problem

$$
\bar{C}_{C L}=\mathbb{E}\left[\max _{\boldsymbol{p}_{p}, \boldsymbol{p}_{d} \in \mathcal{K}} C_{l b}\left(\boldsymbol{p}_{p}, \boldsymbol{p}_{d}\right)\right],
$$

where

$$
\begin{array}{r}
\mathcal{K}=\left\{\boldsymbol{p}_{p}, \boldsymbol{p}_{d} \mid J_{\tau_{d}}\left(\boldsymbol{p}_{p}\right) \leq \epsilon, \mathbf{1}^{T} \boldsymbol{p}_{d}+\mathbf{1}^{T} \boldsymbol{p}_{p} \leq P_{t},\right. \\
\left.\boldsymbol{p}_{p}^{T} \boldsymbol{p}_{d}=0, \boldsymbol{p}_{p} \succeq 0, \boldsymbol{p}_{d} \succeq 0\right\},
\end{array}
$$

where $\bar{C}_{C L}$ is the channel capacity for the closed-loop (CL) system using partially known CSI at the transmitter side, $\mathcal{K}$ is a set such that $\boldsymbol{p}_{p}$ and $\boldsymbol{p}_{d}$ satisfy some constraints, $\boldsymbol{p}_{p}$ and $\boldsymbol{p}_{d}$ represent pilot and data power vectors respectively, $P_{t}$ is the total power, $J_{\tau_{d}}\left(\boldsymbol{p}_{p}\right)$ represents the asymptotic bound for time-delay in (25) or (29) and $\epsilon$ is the minimum accuracy in the estimation, constraint $\mathbf{1}^{T} \boldsymbol{p}_{d}+\mathbf{1}^{T} \boldsymbol{p}_{p} \leq P_{t}$ limits the total power in the design of data and pilots to be smaller than $P_{t}, \boldsymbol{p}_{p}^{T} \boldsymbol{p}_{d}=0$ means that subcarriers used for data transmission cannot be used as pilots which leads to a combinatorial optimization that is not convex. To solve this issue, we use the relaxation approach [10] by omitting the constraint $\boldsymbol{p}_{p}^{T} \boldsymbol{p}_{d}=0$ and solving the relaxed problem. Solving the relaxed problem, one needs only a few subcarriers as pilots with higher amplitudes while the rest are set for data transmission. Finally, $\boldsymbol{p}_{p} \succeq 0$ and $\boldsymbol{p}_{d} \succeq 0$ emphasizes that pilots and data are non-negative values where $\succeq$ is an elementwise operator.

\section{Simulation Results}

In this section we present the simulation results based on the asymptotic bounds. We use the simulation parameters as follows. The number of channel taps $L=4$, number of subcarriers $N=40$, noise power $\sigma^{2}=0.01$, minimum accuracy in time-delay estimation using asymptotic bound is set to $\epsilon=0.001$ and $\epsilon=0.0001$, and a diagonal channel covariance matrix has been used with $\left(R_{h}=\right.$ $\operatorname{diag}([1.8949 ; 1.6222 ; 1.4209 ; 1.9405]))$.

Fig. 1 and Table. I show the asymptotic behavior of the bounds for channel coefficients and time delay estimation respectively. Fig. 1 shows that after 40 subcarriers the asymptotic bound for channel coefficients converges to the non-asymptotic ECRB of channel coefficients. Note that convergence behavior of the asymptotic ECRB of time delay is faster than channel coefficients due to the fact that it decreases by a factor of $N^{3}$ while the asymptotic ECRB of channel coefficients decreases by a factor of $N$. Therefore, to make the difference visible we used the Root Mean Square Error (RMSE) of the difference between asymptotic and non-asymptotic ECRB of time delay. 


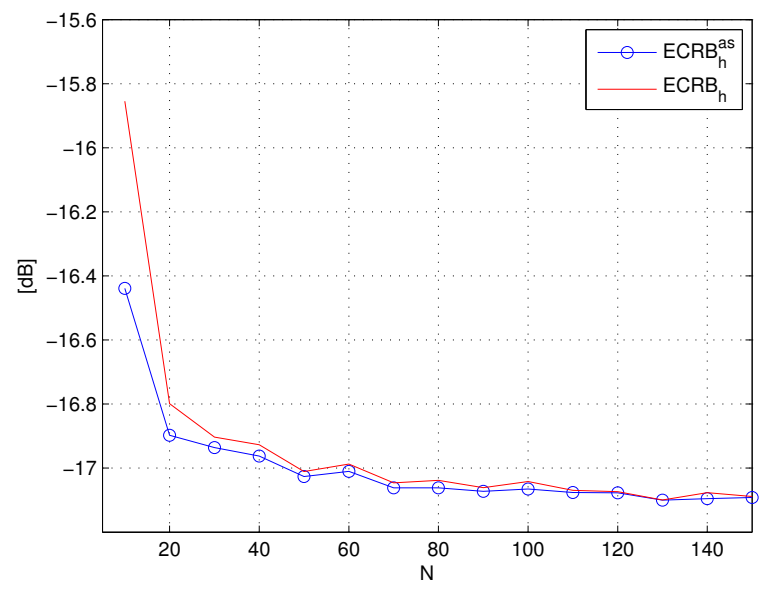

Fig. 1. Asymptotic behavior of $\mathrm{ECRB}_{h}^{a s}$ versus number of subcarriers $N$.

TABLE I

RMSE OF THE DIFFERENCE BETWEEN ASYMPTOTIC AND NON-ASYMPTOTIC ECRB OF TIME-DELAY

\begin{tabular}{|c|c|c|c|c|}
\hline $\mathrm{N}$ & 10 & 50 & 100 & 150 \\
\hline RMSE [dB] & -56.3961 & -86.8914 & -98.9957 & -106.1279 \\
\hline
\end{tabular}

As it is clear from Fig. I the difference is of the order of $-56 d B$ to $-106 d B$ for $N$ from 10 to 150 respectively.

Fig. 2 shows ergodic capacity for closed-loop system $\bar{C}_{C L}$ obtain based on the optimization problem in (37) and (38) versus SNR. From Fig. 2, it is clear that by improving the accuracy in the estimation of time-delay $\bar{C}_{C L}$ is reduced considerably for SNR below $30 d B$ while by increasing SNR the effect of time-delay estimation accuracy on $\bar{C}_{C L}$ is reduced.

\section{CONCLUSION}

Applying asymptotic bounds one can use the simpler expressions of the bounds to the optimization problem specially by increasing the number of subcarriers $N$ and channel taps $L$. In this paper, the performance of near-optimal pilot and data power allocations for the case of asymptotic bounds has been compared with the traditional non-asymptotic bounds. Results show that after a certain number of subcarriers which can be as low as $N=40$, asymptotic bounds converge to the nonasymptotic bounds. Further, the performance of pilot power allocations is only affected negligibly even for the limited number of subcarriers and channel taps.

\section{REFERENCES}

[1] R. Negi and J. Cioffi, "Pilot tone selection for channel estimation in a mobile OFDM system," IEEE Transactions on Consumer Electronics, vol. 44, pp. 1122-1128, Aug. 1998.

[2] I. Barhumi, G. Leus, and M. Moonen, "Optimal training design for MIMO OFDM systems in mobile wireless channels," IEEE Transactions on Signal Processing, vol. 51, no. 6, pp. 1615-1624, Jun. 2003.

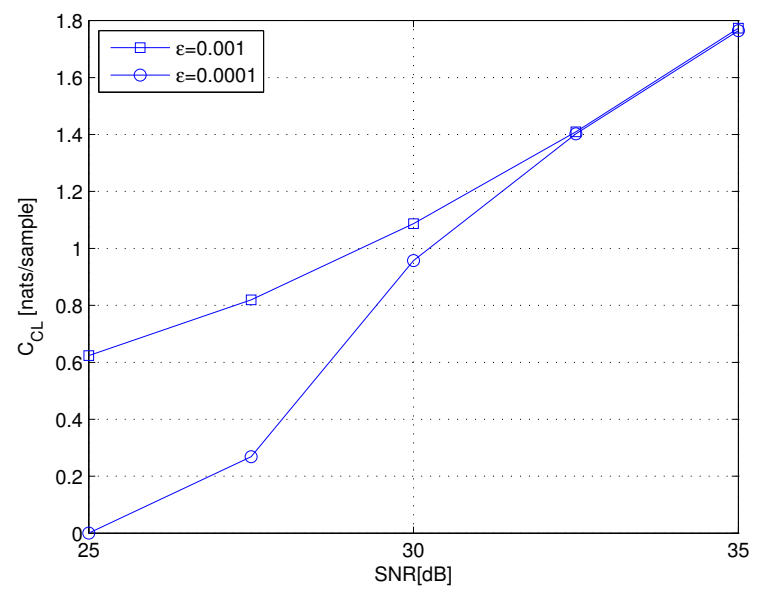

Fig. 2. Ergodic channel capacity for the closed-loop (CL) system using partially known CSI at the transmitter.

[3] H. Minn and N. Al-Dhahir, "Optimal training signals for MIMO-OFDM channel estimation," IEEE Transactions on Wireless Communications, vol. 5, no. 5, pp. 1158-1168, May. 2006.

[4] H. Minn and S. Xing, "An optimal training signal structure for frequency-offset estimation," IEEE Transactions on Communications, vol. 53, no. 2, pp. 343-355, Feb. 2005.

[5] P. Stoica and O. Besson, "Training sequence design for frequency offset and frequency-selective channel estimation," IEEE Transactions on Communications, vol. 51, no. 11, pp. 1910-1917, Nov. 2003.

[6] Tao Cui and C. Tellambura, "Joint channel and frequency offset estimation and training sequence design for MIMO systems over frequency selective channels," in Global Telecommunications Conference, Globecom. 2004, pp. 2344-2348, IEEE.

[7] J. Chen, Y. C. Wu, S. Ma, and T. S. Ng, "Joint CFO and channel estimation for multiuser MIMO-OFDM systems with optimal training sequences," IEEE Transactions on Signal Processing, vol. 56, no. 8, pp. 4008-4019, Aug. 2008.

[8] M.D. Larsen, G. Seco-Granados, and A.L. Swindlehurst, "Pilot optimization for time-delay and channel estimation in OFDM systems," in IEEE International Conference on Acoustics, Speech and Signal Processing (ICASSP). 2011, pp. 3564-3567, IEEE.

[9] R. Montalban, J. A. Lopez-Salcedo, G. Seco-Granados, and A. L. Swindlehurst, "Power allocation approaches for combined positioning and communications ofdm systems," in Proc. IEEE International Workshop on Signal Processing Advances for Wireless Communications (SPAWC). 2013, pp. 694-698, IEEE.

[10] R. Montalban, J. A. Lopez-Salcedo, G. Seco-Granados, and A. L. Swindlehurst, "Power allocation method based on the channel statistics for combined positioning and communications OFDM systems," in IEEE International Conference on Acoustics, Speech and Signal Processing (ICASSP). 2013, pp. 4384-4388, IEEE.

[11] S. Gault, W. Hachem, and P. Ciblat, "Joint sampling clock offset and channel estimation for ofdm signals: Cramer-rao bound and algorithms," IEEE Transactions on Signal Processing, vol. 54, no. 5, pp. 1875-1885, May. 2006.

[12] S. M. Kay, Fundamentals of Statistical Signal Processing: Estimation Theory, Prentice Hall, New York, NY, USA, 2010.

[13] P. Stoica and T. Marzetta, "Parameter estimation problems with singular information matrices," IEEE Transactions on Signal Processing, vol. 49, no. 1, pp. 87-90, Jan. 2001.

[14] B. Hassibi and B. M. Hochwald, "How much training is needed in multiple-antenna wireless links," IEEE Transactions on Information Theory, vol. 49, no. 4, pp. 951-963, Apr. 2003. 